\title{
Research on Gender-related Fingerprint Features, Extracting Fingerprint Features Using Autoencoder Networks for Gender Classification
}

\section{Yong Qi ( $\sim$ qiyong@sust.edu.cn )}

Shaanxi University of Science and Technology

Menzhe Qiu

Shaanxi University of Science and Technology

Huawei Lin

Shaanxi University of Science and Technology

Jiashu Chen

Shaanxi University of Science and Technology

Yanping Li

Shaanxi University of Science and Technology

Hongguang Lei

Shaanxi University of Science and Technology

\section{Research Article}

Keywords: Deep learning, Fingerprint, Gender Identification, 54 Combinations' Testbed, Feature Visualization

Posted Date: March 7th, 2022

DOI: https://doi.org/10.21203/rs.3.rs-1399918/v1

License: (9) This work is licensed under a Creative Commons Attribution 4.0 International License. Read Full License 


\section{Research on Gender-related Fingerprint Features, Extracting fingerprint features using autoencoder networks for gender classification}

Yong Qi ${ }^{1,2^{*}}$, Menzhe Qiu ${ }^{1,2 \dagger}$, Huawei Lin ${ }^{1,2}$, Jiashu Chen ${ }^{1,2}$, Yanping $\mathrm{Li}^{1,2}$ and Hongguang Lei ${ }^{1,2}$

\footnotetext{
"Correspondence:

qiyong@sust.edu.cn

${ }^{1}$ School of Electronic Information and Artificial Intelligence, Shaanxi

University of Science and Technology, 710021 Xi'an, China

${ }^{2}$ Shaanxi Joint Laboratory of Artificial Intelligence, Shaanxi University of Science and

Technology, 710021 Xi'an, China Full list of author information is

available at the end of the article

${ }^{\dagger}$ Equal contributor
}

\begin{abstract}
Background: Fingerprint is an important biological feature of human body, which contains abundant biometric information.At present, the academic exploration of fingerprint gender characteristics is generally at the level of understanding, and the standardization research is quite limited.
\end{abstract}

Methods: A robust approach is presented in this article, Dense Dilated Convolution ResNet Autoencoder, to extract valid gender information from fingerprints. By replacing the normal convolution operations with the atrous convolution in the backbone, prior knowledge is provided to keep the edge details and the global reception field can be extended.

Results: The results were explored from three aspects: 1) The efficiency of the DDC-ResNet. 6 typical automatic feature extraction methods with 9 mainstream classifiers for a total of 54 combinations are evaluated on our dataset and provide unbiased experimental details. Experimental results demonstrate that the combination of our approach outperforms other combinations in terms of average accuracy and separate-gender accuracy. It reaches $96.5 \%$ for average and 0.9752 (males) $/ 0.9548$ (females) for separate-gender accuracy. 2) The effect of fingers. It is found that the best performance of classifying gender with separate fingers is achieved by the right ring finger. 3) The effect of specific features. Based on the observations of the concentrations of fingerprints visualized by our approach, it can be inferred that loops and whorls (level 1), bifurcations(level 2), as well as line shapes (level 3) are connected with gender. Finally, we will open source the dataset that contains 6000 fingerprint images.

Conclusions: The results are demonstrated that autoencoder networks are a powerful method for extracting gender-specific features to help hide the privacy information of the user's gender contained in the fingerprint. Our experiments also identified three levels of features in fingerprints that are important for gender differentiation, including loops and whorls shape, bifurcations shape, and line shapes.

Keywords: Deep learning; Fingerprint; Gender Identification; 54 Combinations' Testbed; Feature Visualization 


\section{Background}

Fingerprint gender identification aims to extract gender-related features from an unidentified fingerprint to recognize one's gender information. It can be divided into two stages, namely extracting as well as classifying [1-8], in which the former step is of great significance since the effectiveness of gender identification, is primarily determined by the sufficiency of gender-related features. Nowadays, classifying ridge-related features extracted manually has achieved fairly good results, reaching an overall accuracy for $90 \%$ for average [8-10]. High performances, however, depends strongly on the manual extraction of features from well-selected regions [11]. These methods have major shortcomings, such as high error, weak robustness, and high labor consumption. So far, with the growing popularity of machine learning and deep learning, automatic feature extraction has become a major foucus.

To realize the automatic feature extraction, considerable work has been done. In machine learning, methods such as DWT, SVD, PCA as well as FFT are extensively used $[3,12,13]$. For deep learning based methods, including deep autoencoder neural networks such as VGG and ResNet,etc have been investigated. [14].

Although numerous algorithms have been proposed, there are still 2 major challenges. First, traditional approaches aquire excessive labor consumption and lack automation. Second, the automatic feature extraction method lacks robustness, which only concerns regions instead of considering the global field. Figure 1 shows the difference between the normal automatic feature extraction method and the deep neural network with global reception consideration. The latter can divide the feature space normatively. Besides, private datasets with disparate data distributions and sizes will directly influence the accuracy,and fingerprint information contains a lot of noise, resulting in low recognition accuracy. Thus, because of the above challenges, we propose a global feature extraction method to improve the efficiency of gender classification.

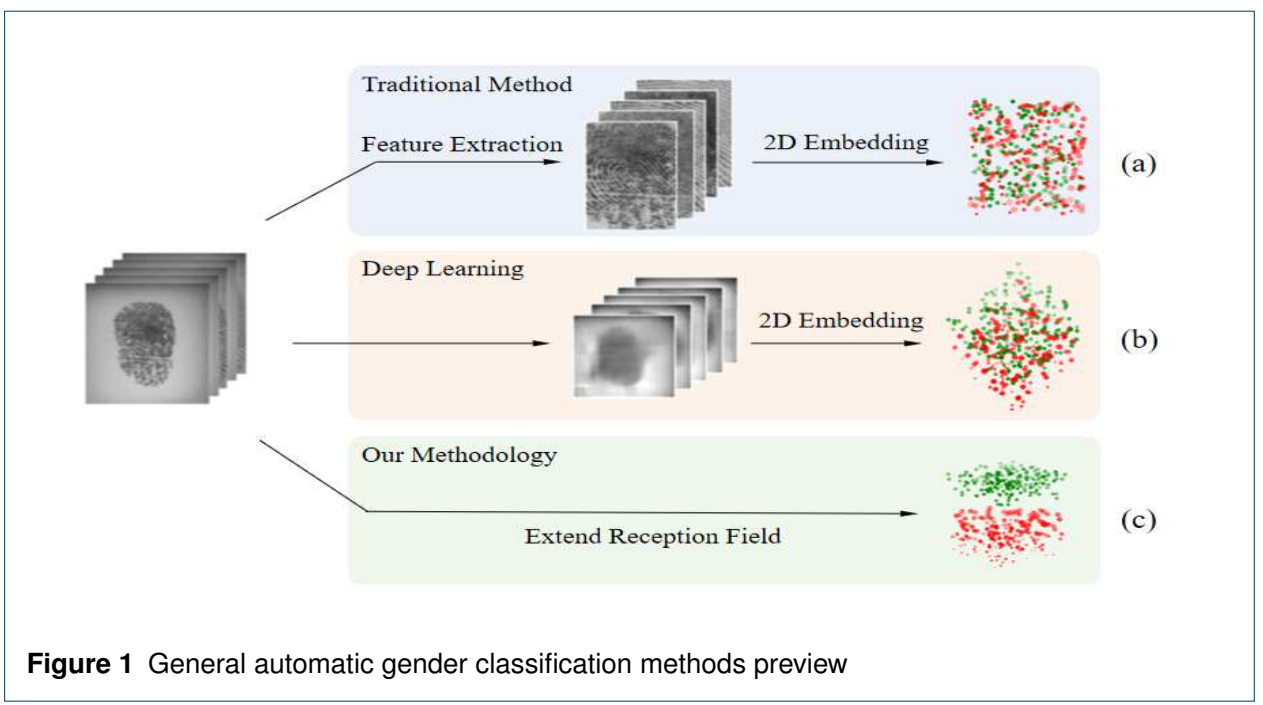

\section{Related Work}

Previous discussions have demonstrated that gender-related features have a significant impact on fingerprint gender identification. In this section, we will first go through the evo- 
lution and then review the progress in recent years in a conclusive table, which is shown in Table 1.

In 1999, Acree [15] manually counted ridges in specific areas on the fingerprint epidermis, showing that ridge count can determine the gender. Then in 2003, the mean epidermal ridge breadth has been proposed to identify gender [11]. Similarly in 2006, Badawi [10] manualy extracted ridge counts, ridge thickness to valley thickness ratio(RTVTR), and white lines count to determine the gender using a neural network as a classifier. Later, Gungadin [16] found a threshold of the ridge density 13 ridges $/ 25 \mathrm{~mm}^{2}$ in 2007 , which determines the gender as male when the ridge density is lower than the threshold. In other words, the female has higher ridge density is probably due to they have lower ridge breadth [11].

Owing to the inevitable error and high manpower consumption of manual feature extractions, automatic fingerprint extraction has been proposed. In 2012, ridge features have been analyzed in the spatial domain using FFT, 2D-DCTT, and PSD [12], reaching an accuracy of $90 \%$ for females and $79.07 \%$ for males, respectively. In the same year, Gnanasivam [3] proposed a method using DWT and SVD as the feature extractor. In 2014, Marasco [13] utilized LBP and LPQ on texture features and used PCA to reduce the features, then the kNN classifier was applied to the extracted features.

In 2017, Gupta [4] applied discrete wavelet transformation to extract fingerprint features, and then output to back propagation neural network for gender classification, and finally obtained $91.45 \%$ classification accuracy. In 2018, Shehu [17] employed CNN models, specifically ResNet-34, were trained using transfer learning technique, to complete the feature extraction for fingerprints, and achieved a combined classification accuracy of $75.20 \%$. Since the presence of the deep convolution autoencoder neural network, deep learning has been widely applied in the feature extraction task especially in the biometric recognition field [14].

\section{Contributions of this paper}

The main contributions of this study are as follows:(1) We propose a feature extraction method of fingerprint that takes global features into account. For existing typical automatic feature extraction methods and classification methods, we make comprehensive comparisons with fair implementation details. (2) We make a comprehensive comparison to test the efficiency of our method. The finger with the richest gender-related features is detected using the highest performance method. Finally, we visualize the concentrations using the selected method, which indicates the regions with the highest contributions and their corresponding specific features in the gender identification task. (3) We will finally open source the dataset since the open-source datasets in fingerprint gender classification are limited.

\section{Experimental Results}

In this section, we first introduce our dataset and adopted implementation details. In the experimental stage, factors that affect gender-related features will be analyzed comprehensively to provide some useful conclusions.

Experimental Setup

\section{Dataset}

The fingerprint dataset used in experiments is obtained from ZK fingerprint acquisition equipment (Figure 3) with 500 dpi, containing 200 persons(102 females and 98 males) and 
Table 1

The overview of the previous methods.

\begin{tabular}{|c|c|c|c|c|c|}
\hline Year & Publisher & $\begin{array}{c}\text { Feature } \\
\text { Extraction }\end{array}$ & Classifier & Results & Dataset \\
\hline 1999 & Acree [15] & $\begin{array}{l}\text { Ridge counting } \\
\text { manually }\end{array}$ & Threshold & $\begin{array}{c}\text { Female ridge } \\
\text { density is } \\
\text { higher }\end{array}$ & $\begin{array}{l}\text { Own dataset } \\
\text { contains } 400 \\
\text { subjects }\end{array}$ \\
\hline 2003 & Kralik [11] & $\begin{array}{c}\text { Mean } \\
\text { epidermal ridge } \\
\text { breadth }\end{array}$ & Threshold & $\begin{array}{l}\text { Ridge breadth } \\
\text { is } 9 \% \text { greater in } \\
\text { males than in } \\
\text { females }\end{array}$ & $\begin{array}{l}\text { Own dataset } \\
\text { contains } 60 \\
\text { subjects }\end{array}$ \\
\hline 2006 & Badawi [10] & $\begin{array}{l}\text { RTVTR, white } \\
\text { line, ridge } \\
\text { thickness }\end{array}$ & $\begin{array}{l}\text { Neural } \\
\text { Network }\end{array}$ & Average $88.8 \%$ & $\begin{array}{l}\text { Own dataset } \\
\text { contains } 220 \\
\text { subjects }\end{array}$ \\
\hline 2007 & Gungadin [16] & $\begin{array}{l}\text { Counting ridges } \\
\text { in the upper } \\
\text { portion of the } \\
\text { radial border }\end{array}$ & Threshold & $\begin{array}{l}\text { Ridge density } \\
\text { of male's } \\
\text { fingerprint tend } \\
\text { to be less than } \\
\text { or equal to } \\
\text { 13ridges } / 25 \mathrm{~mm}^{2}\end{array}$ & $\begin{array}{l}\text { Own dataset } \\
\text { contains } 500 \\
\text { subjects }\end{array}$ \\
\hline 2011 & Arun [9] & $\begin{array}{c}\text { Ridge count, } \\
\text { ridge density, } \\
\text { white line and } \\
\text { RTVTR }\end{array}$ & $\begin{array}{l}\text { SVM with } \\
\text { RBF kernel }\end{array}$ & Overall 96\% & $\begin{array}{c}\text { Own dataset } \\
\text { contains } 150 \\
\text { male and } 125 \\
\text { female images }\end{array}$ \\
\hline 2012 & Kaur [12] & FFT, DCT, PSD & Threshold & $\begin{array}{c}90 \% \text { for female } \\
\& 79.07 \% \text { for } \\
\text { male }\end{array}$ & $\begin{array}{l}\text { Own dataset } \\
\text { contains } 220 \\
\text { subjects }\end{array}$ \\
\hline 2012 & Gnanasivam [3] & DWT+SVD & KNN & $\begin{array}{c}91.67 \% \text { for } \\
\text { male \& } 84.69 \% \\
\text { for female }\end{array}$ & $\begin{array}{l}\text { Own dataset } \\
\text { contains } 357 \\
\text { subjects }\end{array}$ \\
\hline 2014 & Marasco [13] & $\begin{array}{l}\text { LBP and LPQ } \\
\text { descriptor }\end{array}$ & KNN & Overall $88.7 \%$ & $\begin{array}{c}\text { Own dataset } \\
\text { contains } 494 \\
\text { subjects }\end{array}$ \\
\hline 2014 & Gupta [4] & DWT & ANN & Overall $91.45 \%$ & $\begin{array}{l}\text { Own dataset } \\
\text { contains } 55 \\
\text { subjects }\end{array}$ \\
\hline 2016 & Abdullah [1] & $\begin{array}{c}\text { Ridge count, } \\
\text { ridge density, } \\
\text { white line and } \\
\text { RTVTR }\end{array}$ & J48 & Overall $96.28 \%$ & $\begin{array}{l}\text { Own dataset } \\
\text { contains } 296 \\
\text { subjects }\end{array}$ \\
\hline 2016 & Abdullah [18] & $\begin{array}{l}\text { Ridge count, } \\
\text { ridge density, } \\
\text { white line and } \\
\text { RTVTR }\end{array}$ & MLP & Overall $97.25 \%$ & $\begin{array}{l}\text { Own dataset } \\
\text { contains } 300 \\
\text { subjects }\end{array}$ \\
\hline 2017 & Sheetlani [19] & DWT & CNN & Overall $96.60 \%$ & $\begin{array}{l}\text { Own dataset } \\
\text { contains } 80 \\
\text { subjects }\end{array}$ \\
\hline 2017 & $\begin{array}{c}\text { Ashish } \\
\text { Mishra [5] }\end{array}$ & $\begin{array}{l}\text { minutiae, } \\
\text { incipient ridges }\end{array}$ & SVM \& NN & $\begin{array}{l}76.06 \% \text { for } \\
\text { SVM and } 83.7 \\
\% \text { for female }\end{array}$ & NIST \\
\hline 2018 & Wedpathak [8] & $\begin{array}{l}\text { Ridge count \& } \\
\text { RTVTR }\end{array}$ & ANN & $\begin{array}{l}88 \% \text { for male \& } \\
78 \% \text { for female }\end{array}$ & Own dataset \\
\hline 2018 & Shehu [17] & ResNet-34 & CNN & $\begin{array}{c}73.98 \% \text { for } \\
\text { female \& } \\
76.42 \% \text { for } \\
\text { male }\end{array}$ & $\begin{array}{l}\text { Own dataset } \\
\text { contains } 369 \\
\text { subjects }\end{array}$ \\
\hline 2019 & Alam [20] & DWT+SVD & KNN & $\begin{array}{c}91.25 \% \text { for } \\
\text { male \& } 88.96 \% \\
\text { for female }\end{array}$ & $\begin{array}{l}\text { Own dataset } \\
\text { contains } 42 \\
\text { subjects }\end{array}$ \\
\hline 2019 & Rekha [6] & Gabor filter & $\begin{array}{l}\text { KNN, SVM, } \\
\text { Naive } \\
\text { Bayes }\end{array}$ & ror tom & 6onojocio \\
\hline 2021 & $\begin{array}{c}\text { DDC- } \\
\text { ResNet(Ours) }\end{array}$ & Autoencoder & CNN & $\begin{array}{c}\mathbf{9 7 . 5 2} \% \text { for } \\
\text { male \& } \mathbf{9 5 . 4 8} \% \\
\text { for female }\end{array}$ & $\begin{array}{c}\text { Dataset } \\
\text { contains } 200 \\
\text { subjects }\end{array}$ \\
\hline
\end{tabular}


6000 images. Each finger is collected 3 times to guarantee the quality of the fingerprint image.

\section{Implementation Details}

In the preprocessing step, we resize each fingerprint image to 256x256 and normalize it to $[0,1]$. Train : Test ratio is $4: 1$, No repetitive finger is guaranteed in both the training set and test set.

In the feature extraction stage, for DWT, each fingerprint goes through eight levels of decomposition. For SVD, each fingerprint image vector is of size 256. In VGG-Net, we apply the VGG-19 network in which 8 blocks are utilized. Each block contains 2 convolutional layers and a batch of normalization layers. ResNet-18 is applied and each block contains a residual block to prevent the vanishment of gradients. The DDC-ResNet, by replacing the normal convolutional layers with dilated convolutional layers in ResNet, lowers the loss of valid edge features [21]. To keep the fairness, these three types of feature extraction networks are of the vector size $[-1,512]$ in the last layer. Here we set the batch size to be 10 , and the iteration numbers are $10 \mathrm{~K}$. The optimizer we adopt is Adam, in which the learning rate is $3 \times 10^{-4}$. Inspired by Maas [22], the leaky relu is used as the activation function. All the codes are realized with the framework TensorFlow [23] in python. Hardware utilization includes GPU with NVIDIA TITAN Xp, and CPU with $2.8 \mathrm{GHz}, 32 \mathrm{~GB}$ in RAM.

In the classification progress, the Adaboost is used with the n_estimator $=100$, learning rate $=0.01$, max_depth $=7$, and subsample $=1.0$. In SVM, the linear kernel is utilized with the function LinearSVC in the default configuration, $C=100$ and gamma $=0.5$ are set when applying RBF and polynomial kernels. The parameter $\mathrm{k}$ in $\mathrm{kNN}$ is set to be 1 . J48 algorithm is conducted in WEKA software with the default settings [24]. The CNN classifier is comprised of 3 fully connected layers with the leaky relu activation. Classification algorithms except J48 and CNN are all conducted in scikit-learn [25].

\section{Effect of Methods}

To evaluate the effect of different methods on gender-related features, we make a benchmark for the combinations of 6 feature extraction methods coupling with 9 classifiers to compare their average and separate-gender accuracies, as shown in Table 2. In terms of the average accuracy, the DDC-ResNet extractor outperforms other extraction methods when matching with different classifiers. For the classifiers, the Adaboost, SVM with the polynomial kernel, and CNN are more outstanding. On the contrary, the SVM with the linear kernel seems not qualified for the extracted features with high dimensional space in the gender identification task. Regarding the separate-gender performance, most of the results show more correct predictions in males than in females, especially for DWT $+\mathrm{SVM}^{3}$. The difference is more than $10 \%$, which is agreed with the [7,26], suggesting that fingerprints of males contain richer gender-related features in some aspects. To make a more comprehensive analysis, we also evaluate the time consumption of each combination when feeding different batch sizes of data. The result is shown in Table 4. In general, deep learning extractors consume more time than machine learning, and SVD extractor consumes the least time. However, FFT behaves oppositely which is because the transformation does not reduce the feature size. In summary, the combination of DDC-ResNet and CNN outperforms other combinations, reaching an average accuracy of $96.50 \%$ and separate-gender accuracy 
of 0.9743/0.9324. Moreover, males contain richer gender-related features than females. After evaluating the performance of various methods with all test fingers, we will explore how specific fingers influence the gender identification results below.

Table 2

The upper value in each cell represents average accuracy (\%), and the under value shows separate gender accuracy, meaning male accuracy/female accuracy

\begin{tabular}{|c|c|c|c|c|c|c|}
\hline & FFT & DWT & SVD & ResNet & VGG & DDCResNet \\
\hline \multirow{2}{*}{ Adaboost } & 85.280 & 91.494 & 92.096 & 92.068 & 94.138 & 94.253 \\
\hline & $0.8337 / 0.8719$ & $0.9531 / 0.8768$ & $0.9294 / 0.9125$ & $0.9334 / 0.9080$ & $0.9464 / 0.9364$ & $0.9350 / 0.9501$ \\
\hline \multirow{2}{*}{ SVM $^{1}$} & 65.827 & 70.001 & 66.552 & 69.302 & 72.454 & 73.844 \\
\hline & $0.6895 / 0.6270$ & $0.7280 / 0.6720$ & $0.6839 / 0.6471$ & $0.7202 / 0.6658$ & $0.7208 / 0.7283$ & $0.7366 / 0.7403$ \\
\hline \multirow{2}{*}{ SVM $^{2}$} & 82.604 & 88.275 & 86.506 & 85.502 & 88.506 & 89.576 \\
\hline & $0.7935 / 0.8586$ & $0.9144 / 0.8511$ & $0.7910 / 0.9391$ & $0.8210 / 0.8890$ & $0.8192 / 0.9509$ & $0.8260 / 0.9655$ \\
\hline \multirow{2}{*}{ SVM $^{3}$} & 87.310 & 88.735 & 91.609 & 91.713 & 94.713 & 95.333 \\
\hline & $0.8653 / 0.8809$ & $0.9452 / 0.8295$ & $0.9248 / 0.9074$ & $0.9220 / 0.9123$ & $0.9590 / 0.9353$ & $0.9743 / 0.9324$ \\
\hline \multirow{2}{*}{ KNN } & 92.202 & 90.460 & 88.230 & 92.529 & 93.563 & 92.759 \\
\hline & $0.9346 / 0.9094$ & $0.9187 / 0.8905$ & $0.8917 / 0.8729$ & $0.9072 / 0.9434$ & $0.9428 / 0.9285$ & $0.8865 / 0.9687$ \\
\hline \multirow{2}{*}{ J48 } & 73.912 & 90.394 & 76.667 & 90.460 & 88.220 & 89.344 \\
\hline & $0.7224 / 0.7558$ & $0.8977 / 0.9102$ & $0.7296 / 0.8037$ & $0.8979 / 0.9113$ & $0.9195 / 0.8449$ & $0.8921 / 0.8948$ \\
\hline \multirow{2}{*}{ CNN } & 87.220 & 91.360 & 92.413 & 93.333 & 93.678 & 96.500 \\
\hline & $0.8814 / 0.8630$ & $0.9455 / 0.8817$ & $0.9350 / 0.9133$ & $0.9463 / 0.9204$ & $0.9423 / 0.9313$ & $0.9752 / 0.9548$ \\
\hline \multirow{2}{*}{ ID3 } & 78.931 & 90.344 & 89.770 & 90.460 & 90.459 & 91.494 \\
\hline & $0.7682 / 0.8104$ & $0.8727 / 0.9342$ & $0.8856 / 0.9098$ & $0.9149 / 0.8943$ & $0.9099 / 0.8993$ & $0.9159 / 0.9140$ \\
\hline \multirow{2}{*}{ LDA } & 88.498 & 87.192 & 89.721 & 82.106 & 89.385 & 93.599 \\
\hline & $0.9012 / 0.8687$ & $0.8693 / 0.8745$ & $0.9411 / 0.8443$ & $0.7966 / 0.8455$ & $0.9047 / 0.8830$ & $0.9461 / 0.9259$ \\
\hline
\end{tabular}

\section{Effect of Fingers}

After studying the effect of varing methods on gender-related features, we further explore the effect of each finger. We divide the testing fingerprints into 10 sets, each set of which corresponds to a specific finger containing 660 fingerprint images. We apply the DDCResNet coupled with CNN(best performance method above) to test the 10 sets, as listed in Table 3. The result indicates that for each specific finger, the right ring finger(R2) shows the highest accuracy, reaching $92.455 \%$. For 5 pairs of fingers, ring fingers outperform other pairs, reaching an accuracy of $91.413 \%$. For the overall hand, the right hand achieves a higher accuracy than the left hand, which reaches $87.872 \%$. To better understand the effect of fingers on gender-related features, more careful studies will be carried out in the following part.

\section{Effect of Features}

To further research on gender-related features, we apply the grad-cam [27] technique to visualize the heat maps, seeing which part contributes most. Specifically, based on earlier results, we visualize testing fingers by using the DDC-ResNet extractor. The overview of 
Table 3

Estimate performance of each finger(\%), in which $F_{x}$ represents Finger $x$. The index from 1 to 5 means little finger, ring finger, middle finger index finger and thumb respectively. And the $F_{\text {average }}$ represents the average of their fingers.

\begin{tabular}{lcccccc}
\hline & \multicolumn{7}{c}{ Types of Finger $\left(F_{x}\right)$} \\
\cline { 2 - 7 } Hand & $F_{1}$ & $F_{2}$ & $F_{3}$ & $F_{4}$ & $F_{5}$ & $F_{\text {average }}$ \\
\hline Left & 88.291 & 90.371 & 87.152 & 82.819 & 83.455 & 86.418 \\
Right & 85.864 & $\mathbf{9 2 . 4 5 5}$ & 91.363 & 86.455 & 83.224 & $\mathbf{8 7 . 8 7 2}$ \\
Average & 87.078 & $\mathbf{9 1 . 4 1 3}$ & 89.258 & 84.637 & 83.340 & $\backslash$ \\
\hline
\end{tabular}

Table 4

The running time of each combination, each cell means time consumption $(s)$ when feeding $10 / 100 / 1000$ batch size of data.

\begin{tabular}{|c|c|c|c|c|c|c|}
\hline & VGG & ResNet & DDCResNet & DWT & SVD & FFT \\
\hline Adaboost & $3.213 / 3.959 / 19.677$ & $3.776 / 4.213 / 20.581$ & $6.456 / 6.86 / 26.100$ & $0.184 / 2.652 / 18.693$ & $0.165 / 1.786 / 14.445$ & $0.130 / 3.994 / 280.162$ \\
\hline SVM $^{1}$ & $3.176 / 3.905 / 16.530$ & $3.739 / 4.159 / 17.434$ & $6.419 / 6.806 / 22.953$ & $0.160 / 2.013 / 15.674$ & $0.147 / 1.292 / 13.010$ & $0.184 / 3.207 / 48.925$ \\
\hline SVM $^{2}$ & $3.176 / 3.905 / 16.960$ & $3.739 / 4.159 / 17.864$ & $6.419 / 6.806 / 23.383$ & $0.143 / 2.412 / 15.108$ & $0.153 / 1.292 / 12.967$ & $0.029 / 1.688 / 147.075$ \\
\hline SVM $^{3}$ & $3.176 / 3.905 / 16.902$ & $3.739 / 4.159 / 17.806$ & $6.419 / 6.806 / 23.325$ & $0.296 / 1.609 / 16.883$ & $\mathbf{0 . 1 4 6 / 1 . 2 9 1 / 1 2 . 9 4 4}$ & $0.028 / 1.665 / 143.031$ \\
\hline KNN & $3.180 / 3.904 / 16.602$ & $3.743 / 4.158 / 17.506$ & $6.423 / 6.805 / 23.025$ & $0.198 / 1.059 / 18.370$ & $0.162 / 1.294 / 12.889$ & $0.030 / 1.393 / 89.841$ \\
\hline J48 & $3.184 / 3.927 / 16.331$ & $3.747 / 4.181 / 17.235$ & $6.427 / 6.828 / 22.754$ & $0.184 / 1.477 / 15.854$ & $0.148 / 1.296 / 12.791$ & $0.858 / 4.822 / 201.731$ \\
\hline CNN & $3.189 / 3.903 / 15.963$ & $3.752 / 4.157 / 16.867$ & $6.432 / 6.804 / 22.386$ & $0.139 / 1.749 / 17.110$ & $0.156 / 1.300 / 12.756$ & $1.200 / 4.190 / 341.870$ \\
\hline ID3 & $3.176 / 3.920 / 16.629$ & $3.739 / 4.174 / 17.533$ & $6.419 / 6.821 / 23.052$ & $0.142 / 1.842 / 15.556$ & $0.1467 / 1.291 / 12.897$ & $0.062 / 1.756 / 43.519$ \\
\hline LDA & $3.176 / 3.901 / 16.292$ & $3.739 / 4.154 / 17.196$ & $6.419 / 6.801 / 22.715$ & $0.148 / 1.466 / 16.770$ & $0.147 / 1.292 / 12.967$ & $0.138 / 2.478 / 41.830$ \\
\hline
\end{tabular}

visualization is exhibited in Figure 2, It can be seen that concentrations are mainly on or around the center of fingerprints. Detailed observation is shown in Figure 3. The concentration covers the whorl part which belongs to the pattern features of level 1. Further observation reveals that bifurcations are gathered in concentration regions. However, we exclude the sweat pores around the bifurcations since other parts containing more remarkable sweat pores have not received attention. We also find that the concentration regions prefer to be continuous parts. Therefore, it is not hard to say that the foundation of recognition is complete ridges, which explains the reason for manual extraction of minutiae features in well-selected continuous regions.

\section{Conclusion and Future work}

This paper proposes an effective network considering the global reception field in the gender classification task, which is realized by replacing normal convolutions with dilated convolution in the extraction method. The experiment thoroughly explores the efficiency from three aspects. First, comparing our method with various methods with fair implementation details in our dataset. 6 typical automatic feature extraction methods like DWT, SVD, VGG, and our method coupling with 9 mainstream classifiers such as Adaboost, $\mathrm{kNN}, \mathrm{SVM}, \mathrm{CNN}$, etc. a total of 54 combinations are evaluated. Experimental results reveal that the combination of our extractor with the CNN classifier outperforms other combinations. For average accuracy, it reaches $96.50 \%$ and for separate-gender accuracy, it reaches 0.9752(males)/0.9548(females). Second, we investigate the effect of fingers by classifying gender using separate fingers, and find the best-performing finger is the right ring finger, 


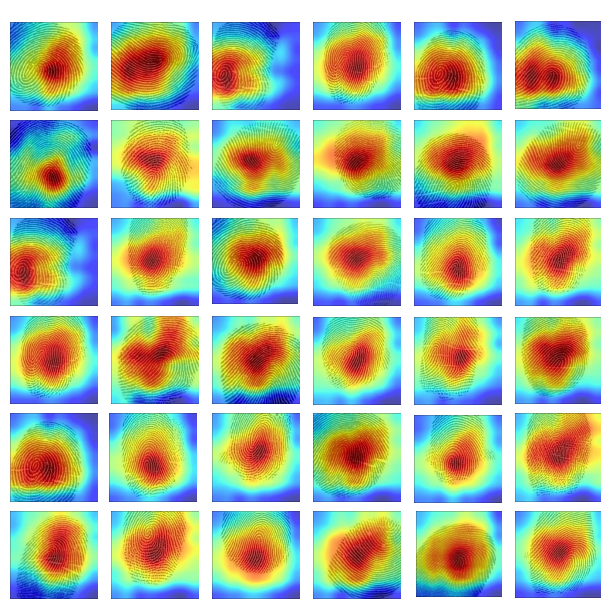

Figure 2 Overall heatmaps

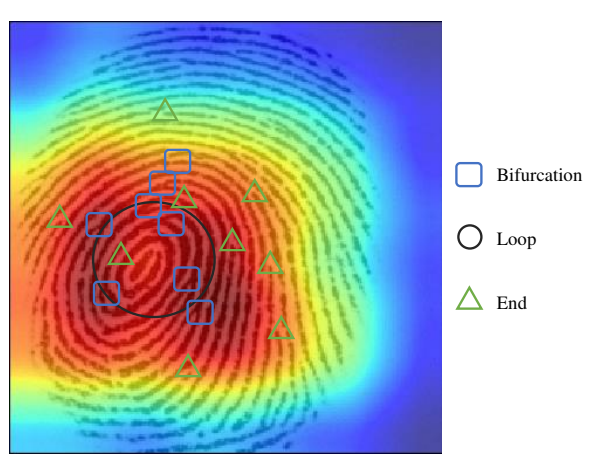

Figure 3 Concentrations in heatmap

which reaches an accuracy of $92.455 \%$. Third, we study the effect of features by visualizing concentrations of fingerprints. Depending on the analysis, loops/whorls (level 1), bifurcations(level 2) and line shapes(level 3) may have a close relationship with gender. This work not only comprehensively explores the efficiency of the proposed method, but also provides a way to observe fingerprint identification features much closer. These specific features will be quantified in the future to further explore the impact on fingerprint recognition.

\section{Overview of Feature Extraction and Classification Algorithms}

In this section, we will introduce our approaches and the implementation principles of automatic feature extraction methods. In addition, we will outline the classification methods utilized in this paper.

\section{Feature Extraction}

\section{Discrete Wavelet Transformation}

Wavelet has been extensively applied in feature extraction, soft-biometrics recognition, and denoising, etc. It decomposes an image into sub-bands containing frequency and orientation information to represent the valid signals. Specifically, a fingerprint image is decomposed into 4 sub-bands at one level, namely low-low(LL), low-high(LH), high-low(HL) 
and high-high(HH) which is shown in Figure 4. Typically, the LL sub-band will be decomposed repeatedly since it is thought to represent the most energy, and $\mathrm{k}$ refers to the repeat times. If $\mathrm{k}$ is set, $\left(3^{*} \mathrm{k}\right)+1$ sub-bands are available. The energy of each sub-band is calculated by equation 1 , which will be used as a feature vector for gender classification $\left(E_{k}\right)$, where $X_{k}(i, j)$ represents the pixel at the position i and $\mathrm{j}$ on the $k t h$ level. W and $\mathrm{H}$ represent the width and the height of the sub-band, respectively.

$$
E_{k}=\frac{1}{W H} \sum_{i=1}^{W} \sum_{j=1}^{H}\left|X_{k}(i, j)\right|
$$

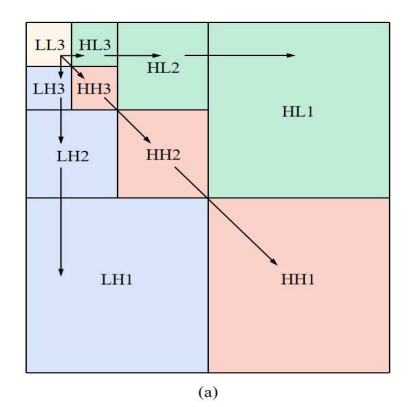

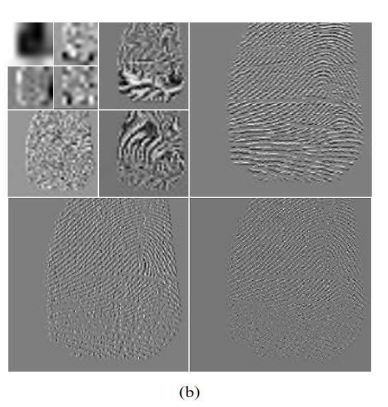

(b)

Figure 4 Discrete Wavelet Transform for the fingers's gender classification

\section{Singular Value Decomposition}

The fundamental of the SVD is that any rectangular matrix can be transformed into the product of three new matrices. Specifically, given a fingerprint image matrix A with $\mathrm{H}$ rows and $\mathrm{W}$ columns, it can be factored into $\mathrm{U}, \mathrm{S}$, and $\mathrm{V}$ by using equation 2, where $U=A A^{T}$ and $V=A^{T} A$ and $\mathrm{S}$ are diagonal matrices that contain the square root eigenvalues with the size of $\mathrm{H}$ by $\mathrm{W}$, which is stored for gender classification.

$$
A=U S V^{T}
$$

\section{Fast Fourier Transform}

The FFT is used to transform a fingerprint image into the frequency domain. The transformed vector contains most of the information in the spatial domain and is used for gender classification. It is presented in equation 3, where $\mathrm{M}$ and $\mathrm{N}$ represent the height and width of the fingerprint image, $\mathrm{k}$ and 1 represent frequency variables, respectively. $0 \leq \mathrm{m}, \mathrm{k} \leq$ $\mathrm{M}-1,0 \leq \mathrm{n}, 1 \leq \mathrm{N}-1$.

$$
F[k, l]=\frac{1}{\sqrt{M N}} \sum_{n=0}^{N-1} \sum_{m=0}^{M-1} f[m, n] e^{-j 2 \pi\left(\frac{m k}{M}+\frac{n l}{N}\right)}
$$

\section{Our method}

Autoencoder neural networks can be divided into the encoder and decoder. Concretely, in the encoder step, the low dimensional data will be compressed into feature vector in high 
dimensional space, and the decoder step is to reconstructs the original data without redundant features. The general equation is described in 4 . where $f$ is the activation function(In this work, we utilized leaky Relu), the $W$ is the parameter matrix, and $b$ is a vector of bias parameters. The feature vector can be optimized by minimizing the distance between the original data and the reconstructed data, and used for gender classification. Our method utilizes ResNet as the backbone, and uses the atrous convolution operation to replace the normal convolutions, as shown in Figure 5. In the block res we utilize atrous rates as 1, 2 and 5 to prevent the gridding effect. [28].And we put the feature vector output from the final convolution layer in the decoder into the classifier for classification.

$$
\begin{aligned}
y & =f(W x+b) \\
x^{\prime} & =f\left(W^{\prime} x+b\right)
\end{aligned}
$$

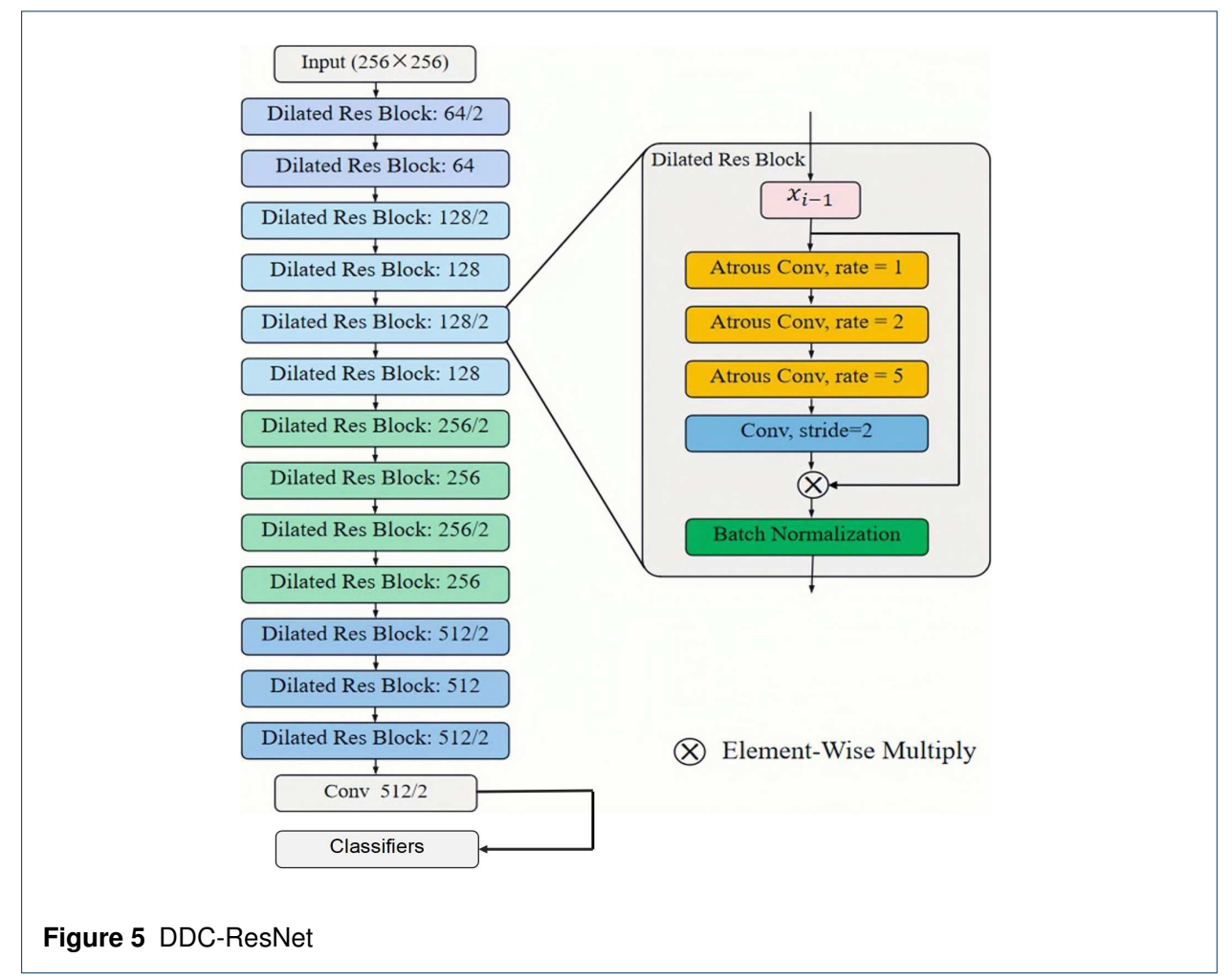

\section{Gender Classification}

To ensure fairness of experiments, we adopt commonly used 9 classifiers in gender classification problem, which are CNN, SVM(3 kernels), kNN, Adaboost, J48, ID3, and LDA. Among them the 3 kernels in SVM refer to linear, radial basis function, and polynomial, respectively. CNN is comprised of fully connected layers. These are all mainstream algorithms used in classification tasks. We threrfore only highlight their implementation details in the experimental result section and more details of the methods are presented in experimental setup section. 


\title{
Gender Classification
}

To ensure fairness of experiments, we adopt commonly used 9 classifiers in gender classification problem, which are CNN, SVM(3 kernels), kNN, Adaboost, J48, ID3, and LDA. Among them the 3 kernels in SVM refer to linear, radial basis function, and polynomial, respectively. CNN is comprised of fully connected layers. These are all mainstream algorithms used in classification tasks. We threrfore only highlight their implementation details in the experimental result section and more details of the methods are presented in experimental setup section.

\section{Acknowledgements}

Not applicable.

Funding

This work is supported by the Research Plan of Shaanxi Provincial Department of Education under Grant 15JK1086, the National Training Program of Innovation and Entrepreneurship for Undergraduates of China under Grant 201910708024, the Shaanxi University of Science and Technology Ph.D. Project under Grant BJ14-07.

\author{
Abbreviations \\ FFT:Fast Fourier Transform;DWT:Discrete Wavelet Transform;VGG:Visual Geometry Group Network;SVM:Support \\ Vector Machine;LDA:Linear Discriminant Analysis;KNN:K-nearest Neighbors;CNN:Convolutional Neural \\ Network;PCA:Principal Component Analysis.
}

Availability of data and materials

The datasets used in the study are available from the corresponding author upon request.

\section{Declarations}

\section{Ethics approval and consent to participate}

The study was approved by Shaanxi University of Science and Technology. All 200 persons who provided fingerprint were fully informed of the purpose and protocol of the study and gave written consent to provide fingerprint information for scientific research and publication only. The authors declare that all experiments were performed according to the ethical principles and the data will not be used in any scenario other than scientific experiments.

\section{Competing interests}

The authors declare that they have no competing interests.

Consent for publication

Not applicable.

\section{Authors' contributions}

QY, QM, LH, CJ and LY contributed to the study concept and design.CJ and LH were involved in the acquisition of data.QY, QM, LH and CJ have contributed to the writing of the manuscript. All authors read and approved the final manuscript.

\section{Author details}

${ }^{1}$ School of Electronic Information and Artificial Intelligence, Shaanxi University of Science and Technology, 710021 Xi'an, China. ${ }^{2}$ Shaanxi Joint Laboratory of Artificial Intelligence, Shaanxi University of Science and Technology, 710021 Xi'an, China

\section{References}

1. Abdullah, S., Rahman, A., Abas, Z., Saad, W.: Fingerprint gender classification using univariate decision tree (j48). Network (MLPNN) 96(95.27), 95-95 (2016)

2. Abdullah, S.F., Rahman, A., Abas, Z., Saad, W.: Support vector machine, multilayer perceptron neural network, bayes net and k-nearest neighbor in classifying gender using fingerprint features. International Journal of Computer Science and Information Security 14(7), 336 (2016)

3. Gnanasivam, P., Muttan, D.S.: Fingerprint gender classification using wavelet transform and singular value decomposition. arXiv preprint arXiv:1205.6745 (2012)

4. Gupta, S., Rao, A.P.: Fingerprint based gender classification using discrete wavelet transform \& artificial neural network. International Journal of Computer Science and mobile computing 3(4), 1289-1296 (2014)

5. Mishra, A., Maheshwary, P.: A novel technique for fingerprint classification based on naive bayes classifier and support vector machine. International Journal of Computer Applications 975, 8887 (2017)

6. Rekha, V., Gurupriya, S., Gayadhri, S., Sowmya, S.: Dactyloscopy based gender classification using machine learning. In: 2019 IEEE International Conference on System, Computation, Automation and Networking (ICSCAN), pp. 1-5 (2019). IEEE

7. Shinde, M.K., Annadate, S.: Analysis of fingerprint image for gender classification or identification: using wavelet transform and singular value decomposition. In: 2015 International Conference on Computing Communication Control and Automation, pp. 650-654 (2015). IEEE 
8. Wedpathak, G.S., Kadam, D., Kadam, K., Mhetre, A., Jankar, V.: Fingerprint based gender classification using ann. International Journal of Recent Trends in Engineering \& Research (IJRTER) 4(3), 4 (2018)

9. Arun, K., Sarath, K.: A machine learning approach for fingerprint based gender identification. In: 2011 IEEE Recent Advances in Intelligent Computational Systems, pp. 163-167 (2011). IEEE

10. Badawi, A.M., Mahfouz, M., Tadross, R., Jantz, R.: Fingerprint-based gender classification. IPCV 6(8), (2006)

11. Kralik, M., Novotny, V.: Epidermal ridge breadth: an indicator of age and sex in paleodermatoglyphics. Variability and evolution 11(2003), 5-30 (2003)

12. Kaur, R., Mazumdar, S.G.: Fingerprint based gender identification using frequency domain analysis. International Journal of Advances in Engineering \& Technology 3(1), 295 (2012)

13. Marasco, E., Lugini, L., Cukic, B.: Exploiting quality and texture features to estimate age and gender from fingerprints. In: Biometric and Surveillance Technology for Human and Activity Identification XI, vol. 9075, p. 90750 (2014). International Society for Optics and Photonics

14. Chen, M., Shi, X., Zhang, Y., Wu, D., Guizani, M.: Deep features learning for medical image analysis with convolutional autoencoder neural network. IEEE Transactions on Big Data (2017)

15. Acree, M.A.: Is there a gender difference in fingerprint ridge density? Forensic science international 102(1), 35-44 (1999)

16. Sudesh Gungadin, M.: Sex determination from fingerprint ridge density. Internet Journal of Medical Update 2(2) (2007)

17. Shehu, Y.I., Ruiz-Garcia, A., Palade, V., James, A.: Detailed identification of fingerprints using convolutional neural networks. In: 2018 17th IEEE International Conference on Machine Learning and Applications (ICMLA), pp. 1161-1165 (2018). IEEE

18. Abdullah, S., Rahman, A., Abas, Z., Saad, W.: Multilayer perceptron neural network in classifying gender using fingerprint global level features. Indian Journal of Science and Technology 9(9), 1-6 (2016)

19. Sheetlani, J., Pardeshi, R., et al.: Fingerprint based automatic human gender identification. Int. J. Comput. App 170(7), 1-4 (2017)

20. Alam, S., Dua, M., Gupta, A., et al.: A comparative study of gender classification using fingerprints. In: 2019 6th International Conference on Computing for Sustainable Global Development (INDIACom), pp. 880-884 (2019). IEEE

21. Wang, P., Chen, P., Yuan, Y., Liu, D., Huang, Z., Hou, X., Cottrell, G.: Understanding convolution for semantic segmentation. In: 2018 IEEE Winter Conference on Applications of Computer Vision (WACV), pp. 1451-1460 (2018). IEEE

22. Maas, A.L., Hannun, A.Y., Ng, A.Y., et al.: Rectifier nonlinearities improve neural network acoustic models. In: Proc. Icml, vol. 30, p. 3 (2013). Citeseer

23. Abadi, M., Agarwal, A., Barham, P., Brevdo, E., Chen, Z., Citro, C., Corrado, G.S., Davis, A., Dean, J., Devin, M., et al.: Tensorflow: Large-scale machine learning on heterogeneous distributed systems. arXiv preprint arXiv:1603.04467 (2016)

24. Holmes, G., Donkin, A., Witten, I.H.: Weka: A machine learning workbench. In: Proceedings of ANZIIS'94-Australian New Zealnd Intelligent Information Systems Conference, pp. 357-361 (1994). IEEE

25. Pedregosa, F., Varoquaux, G., Gramfort, A., Michel, V., Thirion, B., Grisel, O., Blondel, M., Prettenhofer, P., Weiss, R., Dubourg, V., et al.: Scikit-learn: Machine learning in python. the Journal of machine Learning research 12, 2825-2830 (2011)

26. Gnanasivam, P., Vijayarajan, R.: Gender classification from fingerprint ridge count and fingertip size using optimal score assignment. Complex \& Intelligent Systems 5(3), 343-352 (2019)

27. Selvaraju, R.R., Cogswell, M., Das, A., Vedantam, R., Parikh, D., Batra, D.: Grad-cam: Visual explanations from deep networks via gradient-based localization. In: Proceedings of the IEEE International Conference on Computer Vision, pp. 618-626 (2017)

28. Yu, F., Koltun, V.: Multi-scale context aggregation by dilated convolutions. arXiv preprint arXiv:1511.07122 (2015) 